\title{
Investigating the uniqueness of Singular Values for Image Recognition
}

\author{
Nesreen Nusair ${ }^{1}$ and Moh'd Belal Al-Zoubi ${ }^{2}$ \\ ${ }^{1}$ Computer Engineering, German Jordan University \\ Amman, Jordan \\ ${ }^{2}$ Computer Information System, Jordan University \\ Amman, Jordan
}

\begin{abstract}
It has been reported that the Singular Values in the S matrix of the Singular Value Decomposition (SVD) is unique. This allows a reduction in the amount of processing needed to recognize the images by identifying the images based on a few unique values instead of comparing all of the image values.

SVD is a method that transforms matrix A into product USVT where $\mathrm{U}$ and $\mathrm{V}$ transpose as the orthogonal matrices and $\mathrm{S}$ is the diagonal matrix and allows the refactoring of a digital image into three matrices. Using the Singular Values of such refactoring allows representation of the image with a smaller set of values to preserve useful features of the original image. The need to find more effective techniques to recognize images using the least resources possible by finding distinguished unique values is always required because of available resource limitations.

In this paper, the uniqueness of the Singular Values is investigated when applied in image recognition. The study includes applying the SVD on gray images, and of those, mainly on two types of images known as identical and distorted images. The distortions on the images include noise, scaling the images by half and rotating the by $45^{\circ}$.
\end{abstract}

Keywords: Singular Value Decomposition (SVD), Singular Values, Euclidean Distance.

\section{Introduction}

The resources needed to process thousands of images are exponentially huge, so the need to find a technique that recognizes images based on distinguished unique values becomes a necessity. Using only few unique values can reduce the number of comparisons from thousands of values to merely a few values. This reduction will also reduce the needed resources to recognize the images. SVD is a method that transforms matrix A into the product of three matrices, where $\mathrm{A}$ is a matrix with the size of mxn. the matrix A equals the product of $U_{m x m}$ matrix and $S_{m x n}$ martix and the $\mathrm{V}^{\mathrm{T}}$ matrix. Which allows the refactoring of a digital image into three matrices where $\mathrm{U}$ and $\mathrm{V}$ transpose as the orthogonal matrices and $S$ is the diagonal matrix. [1] Using the Singular Values of such refactoring allows the representation of the image with a smaller set of values and the preservation of useful features of the original image. SVD is an attractive algebraic transformation in image processing because of its limitless advantages such as maximum energy packing (usually used in compression), the ability to manipulate the image in base of two distinctive subspaces data and noise subspaces (usually used in noise filtering and watermarking applications). Also it is usually used in the solving of the least squares problem, computing pseudo- inverse of a matrix and multivariate analysis. SVD is a robust and reliable orthogonal matrix decomposition method due to its conceptual and stability motives becoming more and more popular in signal processing area. SVD has the ability to adapt to the variations in the local statistics of an image. [2]

In this paper, the Singular Values uniqueness, effectiveness and the minimal number of values needed to recognize the identical and distorted images are investigated. The study mainly relies on verifying and to what distinction the Singular Values can recognize images by applying SVD on gray images to test its capability and the studied types of distortions used are Gaussian noise, Salt and Pepper noise, half-size, and a rotation of $45^{\circ}$.

\section{Overview of SVD}

SVD is a linear algebra that transforms matrix A to produce $\mathrm{USV}^{\mathrm{T}}$ matrices that allow the factoring of the digital image. Figure 1 ("Factoring A to USV'") illustrates the factorization of matrix A into three matrices: [1] 


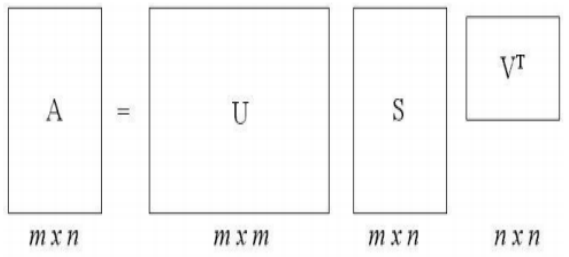

Fig 1 Factoring A to USV ${ }^{\mathrm{T}}$.

The SVD theorem states that: [1]

$\mathrm{A}=\mathrm{USV}^{\mathrm{I}}$

Amxn $=$ Umxm Smxn $V^{\mathbb{I}}$ nxn

$\mathrm{U}$ Matrix is an $\mathrm{m} \times \mathrm{m}$ orthogonal matrix, the Column vectors $\mathrm{u}_{\mathrm{i}}$, for $\mathrm{i}=1,2, \ldots, \mathrm{m}$, form an orthonormal set: [1]

$\mathbb{U}=\left[\mathrm{u}_{1}, \mathrm{u}_{2}, \ldots, \mathrm{u}_{\mathrm{T}}, \mathrm{u}_{\mathrm{T}+1}, \ldots, \mathrm{u}_{\mathrm{m}}\right]$

$\mathrm{u}_{\mathrm{i}}^{\mathrm{T}} \mathrm{u}_{\mathrm{j}}=\delta_{\mathrm{ij}}=\left\{\begin{array}{l}1_{x v x w} \tilde{\mathrm{i}}=\mathrm{j} \\ 0_{x v x} \tilde{\mathrm{i}} \neq \mathrm{j}\end{array}\right.$

$\mathrm{V}$ matrix is an $\mathrm{n} \times \mathrm{n}$ orthogonal matrix, column vectors $\mathrm{v}_{\mathrm{i}}$ for $\mathrm{i}=$ $1,2, \ldots, \mathrm{n}$, form an orthonormal set: [1]

$\mathrm{V}=\left[\mathrm{V}_{1}, \mathrm{~V}_{2}, \ldots, \mathrm{V}_{\mathrm{r}}, \mathrm{V}_{\mathrm{r}+1} \ldots, \mathrm{V}_{\mathrm{n}}\right]$

$\mathrm{v}_{\mathrm{i}}^{\mathrm{T}} \mathrm{V}_{\mathrm{j}}=\delta_{\mathrm{ij}=}=\left\{\begin{array}{l}1_{x x w} \mathrm{i}=\mathrm{j} \\ 0_{x x w} \mathrm{i} \neq \mathrm{j}\end{array}\right.$

$\mathrm{S}$ is an $\mathrm{m} \times \mathrm{n}$ diagonal matrix with Singular Values $(\mathrm{S})$ on the diagonal: [1]

$$
S=\left[\begin{array}{ccccccc}
\sigma_{1} & 0 & \cdots & 0 & 0 & \cdots & 0 \\
0 & \sigma_{2} & \cdots & 0 & 0 & \cdots & 0 \\
\vdots & \vdots & \ddots & \vdots & \vdots & \ddots & \vdots \\
0 & 0 & \cdots & \sigma_{r} & 0 & \cdots & 0 \\
0 & 0 & \cdots & 0 & \sigma_{r+1} & \cdots & 0 \\
\vdots & \vdots & \ddots & \vdots & \vdots & \ddots & \vdots \\
0 & 0 & \cdots & 0 & 0 & \cdots & \sigma_{n} \\
0 & 0 & \cdots & 0 & 0 & \cdots & 0
\end{array}\right]
$$

Fig 2 Matrix S.

For $\mathrm{i}=1,2, \ldots, \mathrm{n}$, ,i are called Singular Values (SV) of matrix A. The $\mathrm{v}_{\mathrm{i}}$ 's and $\mathrm{u}_{\mathrm{i}}$ 's are called right and left singular-vectors of A: [1]

$\sigma_{1} \geq \sigma_{2} \geq \cdots \times \sigma_{\mathrm{r}}>0_{2}$ and $\sigma_{\mathrm{r}+1}=\sigma_{\mathrm{r}+2}={ }^{\mathrm{x}}=\sigma_{\mathrm{N}}=0(7)$

\subsection{Properties of SVD}

There are many properties and attributes of SVD. Here part of the properties is presented [1]:

- The Singular Values on the diagonal of S matrix are unique; however, matrices $\mathrm{U}$ and $\mathrm{V}$ are not unique.

- Matrix A rank equals the nonzero Singular Values number.
- $\quad$ Since $\mathrm{A}^{\mathrm{T}} \mathrm{A}=\mathrm{VS}^{\mathrm{T}} \mathrm{SV}^{\mathrm{T}}$, so $\mathrm{V}$ diagonalizes $\mathrm{A}^{\mathrm{T}} \mathrm{A}$, it follows that the $\mathrm{v}_{j} \mathrm{~S}$ are the eigenvector of $\mathrm{A}^{\mathrm{T}} \mathrm{A}$.

- $\quad$ Since $\mathrm{AA}^{\mathrm{T}}=\mathrm{USS}^{\mathrm{T}} \mathrm{U}^{\mathrm{T}}$, it follows that $U$ diagonalizes $\mathrm{AA}^{\mathrm{T}}$ and that the $\mathrm{u}_{\mathrm{i}}$ 's are the eigenvectors of $\mathrm{AA}^{\mathrm{T}}$.

- If $A$ has rank of $r$ then $v_{j}, v_{j}, \ldots, v_{r}$ form an orthonormal basis for range space of $\mathrm{A}^{\mathrm{T}}$, $R\left(A^{T}\right)$, and $u_{j}, u_{j}, \ldots, u_{r}$ form an orthonormal basis for range space $A, R(A)$.

\subsection{Objective of the Project}

During the last few years, processing thousands of images has required an enormous amount of resources and making comparisons has also become a challenge in a huge dataset. This can be solved by finding more effective techniques to recognize images using distinguished unique values. SVD is a robust and reliable orthogonal matrix decomposition method due to its conceptual and stability motives becoming more and more popular in the signal processing area. SVD has the ability to adapt to the variations in the local statistics of an image and its properties. One of the properties is the uniqueness of the Singular Values in the S matrix which allows for reduction in the amount of processing needed to recognize the images by identifying the images based on a few unique values instead of comparing all of the image values.

\section{Proposed work}

The Singular Values uniqueness, effectiveness and the minimal number of values needed to recognize the images are investigated. The paper includes applying the singular value decomposition (SVD) on gray identical and distorted images. The distortions on the images include noise, scaling the images by half, and a rotation of $45^{\circ}$.

It has been reported that the Singular Values in the S matrix of the SVDs are unique, and based on that, we extracted and stored the Singular Values for each image. Then in order to extract the perfect match image (identical image / nearest image) we calculated the Euclidean Distance between the original image and (the original dataset's images and the distorted dataset's images). Each dataset used in this research included the original images dataset and the distorted images dataset. We added the distortions on all of the images included on the original dataset to create the distorted images datasets. The distorted images datasets include a Salt and Pepper noised dataset, a Gaussian noised dataset, a $45^{\circ}$ rotated dataset, and half-sized dataset.

\subsection{Datasets}

In this study, we used three datasets: The Africans Dataset [3], the Airplanes Dataset [3] and the Shapes Dataset [4]. 
The first one is the Africans Dataset. This data set was used in statistical moments based on noise classification using feedforward, backward-propagation neural network research [3]. This dataset contains 1000 original images, and 4000 distorted images were created from the original images by adding Salt and Pepper noise, Gaussian noise, $45^{\circ}$ rotation, and half-size scaling.

The second Dataset is the Airplane Dataset which contains 800 original images. 3200 distorted images from the original images by adding Salt and Pepper noise, Gaussian noise, $45^{\circ}$ rotation and, half-size scaling were created.

The third Dataset is the Shapes Dataset. This dataset contains 799 original images. 3196 distorted images from the original images by adding Salt and Pepper noise, Gaussian noise, $45^{\circ}$ rotation, and half-size scaling were created.

\subsection{Steps to conduct image recognition using SVD}

In this study, the uniqueness of the Singular Values when applied in image recognition is investigated. also the effectiveness and the minimal number of values needed to recognize the images are investigated as well. It has been reported that the Singular Values in the S matrix of the SVDs are unique. These unique values can reduce the number of comparisons from thousands of values to just a few values. The significant improvement is shown when reducing the number of comparisons, and the needed resources are also reduced. Below, Figure 3 ("Used methodology") shows the different stages carried out in this research:



Fig 3 Used methodology.

1) Calculate SVD for Image, SVD transforms matrix A into product $\mathrm{USV}^{\mathrm{T}}$ where $\mathrm{U}$ and $\mathrm{V}$ are orthogonal matrices and $\mathrm{S}$ is a diagonal matrix. The Singular Values are on the diagonal in the $\mathrm{S}$ matrix.
2) Extract Singular Values for each image from the diagonal of the $\mathrm{S}$ matrix.

3) Store Singular Values for each image.

4) To extract the perfect match image/nearest image we calculate the Euclidean Distance between the original image and (the original dataset's images and the distorted dataset's images).

5) Compare the calculated Euclidean Distance with the other images.

6) Extract the perfect match/nearest image by finding the lowest Euclidean Distance value for the image and its identical/nearest image.

\section{Experimental results}

Table 1 illustrates the result's summary for the three datasets. The datasets are the Africans dataset, the Airplanes dataset and the Shapes dataset.

The recognition rate for each result are calculated based on the percentage of the number of the recognized images to the total number of images in the dataset. Eq. (8) illustrates the used equation Where $\mathrm{X}$ is the number of the recognized images and $\mathrm{Y}$ is the total number of the images in the dataset:

Recognition rate $=\mathrm{X} / \mathrm{Y} * 100 \%$

All identical images are successfully recognized with a $100 \%$ recognition rate on all three datasets using only one Singular Value. This result is attained due to the fact that the Singular Values of the SVDs are unique. By only relying on one Singular Value the images can be matched.

Gaussian noised images show extreme differences. The results are $95.7 \%, 59.5$ and $39.8 \%$ for the Africans dataset, the Airplanes dataset and the Shapes dataset, respectively. The difference in the results is because Gaussian noise makes major changes on the values of the image's matrix. Because Gaussian noise effects each pixel in the original image generating the noisy image with snowy appearance. This means that each pixel in the noisy image is the sum of the pixel value in the original image with a random Gaussian distributed noise value. which makes major changes on the images' values.

Salt and Pepper images result are consistent with (79.7\%, 70\% and $63.95 \%$ ), respectively. Salt-and-pepper noise is a noise that presents itself as sparsely occurring white and black pixels. The consistency in the results is because Salt and Pepper noise makes relatively fewer changes to the values of the image's matrix than Gaussian noise.

As for the $45^{\circ}$ rotated images and the scaled images, the recognition rates are extremely low. The recognition rate's achieved value is between $0.1 \%$ and $3.1 \%$. The difference in the 
results is because rotation changes the coordinates for the pixels which changes the image matrix. Also Scaling changes, the location and the values of the image's matrix because it changes the size of the image and the value of the pixels.

\begin{tabular}{|c|c|c|c|}
\multicolumn{5}{|c}{ Table 1. Results summary } \\
\hline $\begin{array}{c}\text { Dataset } \\
\text { Result }\end{array}$ & $\begin{array}{c}\text { Africans } \\
\text { Dataset } \\
\text { Recognition } \\
\text { Rate) }\end{array}$ & $\begin{array}{c}\text { Airplanes } \\
\text { Dateset } \\
\text { Recognition } \\
\text { Rate) }\end{array}$ & $\begin{array}{c}\text { Shapes } \\
\text { Dataset } \\
\text { (Recognition } \\
\text { Rate) }\end{array}$ \\
\hline $\begin{array}{c}\text { Identical } \\
\text { images } \\
\text { (1 Singular } \\
\text { Value) }\end{array}$ & $100 \%$ & $100 \%$ & $\begin{array}{c}100 \% \\
\text { (18 extra } \\
\text { identical } \\
\text { images } \\
\text { found) }\end{array}$ \\
\hline $\begin{array}{c}\text { Identical } \\
\text { images } \\
\text { (2 Singular } \\
\text { Values) }\end{array}$ & - & - & $100 \%$ \\
\hline $\begin{array}{c}\text { Gaussian } \\
\text { noised } \\
\text { images } \\
\text { (15 Singular } \\
\text { Values) }\end{array}$ & $95.7 \%$ & $59.5 \%$ & $39.8 \%$ \\
\hline $\begin{array}{c}\text { Salt \& } \\
\text { Pepper } \\
\text { noised } \\
\text { images } \\
(12 \text { Singular } \\
\text { Values) }\end{array}$ & $79.7 \%$ & $70 \%$ & $63.95 \%$ \\
\hline $\begin{array}{c}45 \text { rotated } \\
\text { images } \\
(15 \text { Singular } \\
\text { Values) }\end{array}$ & $3.1 \%$ & $0.25 \%$ & $3.1 \%$ \\
\hline $\begin{array}{c}\text { Scaled } \\
\text { images } \\
(15 \text { Singular } \\
\text { Values) }\end{array}$ & $0.1 \%$ & $0.1 \%$ & $0.13 \%$ \\
\hline
\end{tabular}

\section{Conclusion and future work}

\subsection{Conclusion}

In this study, the uniqueness of the Singular Values when applied to image recognition is investigated. Also the effectiveness and the minimal number of values needed to recognize the images is investigated.

The Singular Values are unique and can be used to recognize images. The SVD works best when the images are identical with $100 \%$ recognition rate using only one Singular Value, and does not work well on rotation and scaling. Gaussian noised images show extreme variance between the dataset's results. On the other hand, Salt and Pepper noised images results are consistent between the datasets.

Gaussian noised images results have a high of $95 \%$ and a low of $39 \%$ recognition rate. These results are using 15 Singular Values. The recognition rate was low for singular values less than 15 . As for the Salt and Pepper results, they are close with the high of $79 \%$ and the low of $63 \%$ recognition rate using 12 Singular
Values. The recognition rate was low for singular values less than 12 . The $45^{\circ}$ rotated images and the scaled images recognition rates are extremely low. The recognition rate's achieved values are between $0.1 \%$ and $3.1 \%$.

The Singular Values can be used to recognize identical images safely with recognition rate of $100 \%$ using just one Singular Value while using this technique to recognize noised images depends on the application and the accuracy needed. As for the rotated and scaled images, SVD can't be used due to the low recognition rate.

\subsection{Future Work}

This research has investigated the uniqueness of Singular Values and its potential to be used to recognize images. also found the minimal number of Singular Values to recognize the identical and distorted images.

This future work section presents some of the work that needs to be done to be able to verify even more on the potentials of using the SVD to recognize the images. This can be achieved by testing the SVD's capabilities by using more datasets. Additionally, testing this technique on colored images to investigate the capabilities of the SVD on recognizing colored images.

\section{References}

[1] Lijie Cao, Singular Value Decomposition Applied to Digital Image Processing, Division of Computing Studies, Arizona State University Polytechnic Campus, Mesa, Arizona State University Polytechnic Campus, 2006.

[2] Rowayda Sadek, SVD Based Image Processing Applications: State of The Art, Contributions and Research Challenges, International Journal of Advanced Computer Science and Applications (IJACSA), Vol. 3, No. 7, 2012.

[3] James Wang. (2008). Research Group. http://wang.ist.psu.edu/docs/home.shtml.

[4] LonginLatecki. (2015). Temple University. http://www.cis.temple.edu.

[5] Tat-Jun Chin, Konrad Schindler and David Suter, Incremental Kernel SVD for Face Recognition with Image Sets, 7th IEEE Conf. Automatic Face and Gesture Recognition, 2006.

[6] Daoqiang Zhang, Songcan Chen and Zhi-Hua Zhou, A New Face Recognition Method based on SVD Perturbation for Single Example Image per Person, Applied Math. and Computation, vol. 163, no. 2, 2005.

[7] Awwal Rufai, Gholamreza Anbarjafari and Hasan Demirel, Lossy image compression using singular value decomposition and wavelet difference reduction, Digital Signal Processing, vol. 24, pp. 117-123, 2014. 
[8] Ori Bryt and Michael Elad, Compression of facial images using the K-SVD algorithm, The Electrical Engineering Department,Journal of Visual Communication and Image Representation, 19:270-282, 2008.

[9] Patrick Wa1demar and Tor Ramstad, Hybrid KLT-SVD Image Compression, IEEE International Conference on Acoustics, Speech, and Signal, Munich, 4, 1997.

[10] Yanmin He, Tao Gan, Wufan Chen and Houjun Wang, Adaptive Denoising by Singular Value Decomposition, IEEE Signal Process. Lett., vol. 18, no. 4, 2011.

[11] Ajit Rajwade, Anand Rangarajan and Arunava Banerjee, Image Denoising Using the Higher Order Singular Value Decomposition ,IEEE Trans. Pattern Anal. Mach. Intell., vol. 35, no. 4, 2013.

[12] Chih-Chin Lai, A digital watermarking scheme based on singular value decomposition and tiny genetic algorithm, Digital Signal Process., vol. 21, pp. 522-527, 2011.

[13] Nasrin Makbol and Bee Khoo, Robust blind image watermarking scheme based on Redundant Discrete Wavelet Transform and Singular Value Decomposition, Int. J. Electron. Commun. (AEU) 67(2), 102-112,2013.

[14] Saeed Rastegar Fateme Namazi, Khashayar Yaghmaie and Amer Aliabadian, Hybrid watermarking algorithm based on Singular Value Decomposition and Radon transform ,International Journal of Electronics and Communications (AEÜ) 65, 658-663, 2011.

[15] Michel Marie Deza and Elena Deza, Encyclopedia of Distances, Springer, p. 94, 2009.

[16] Phillip Poon, Wei-Ren Ng and Varun Sridharan, Image Denoising with Singular Value Decomposition and Principal Component Analysis, 2009.

[17] Deepa Kanmani, SVD based Image Watermarking Scheme, IJCA Special Issue on Evolutionary Computation for Optimization Techniques, 2010.

[18] Kuo-Liang Chung, Wei-Ning Yang, Yong-Huai Huang, Shih-Tung $\mathrm{Wu}$ and Yu-Chiao Hsu, On SVD-based watermarking algorithm, Applied Mathematics and Computation, 54-57,2007. 